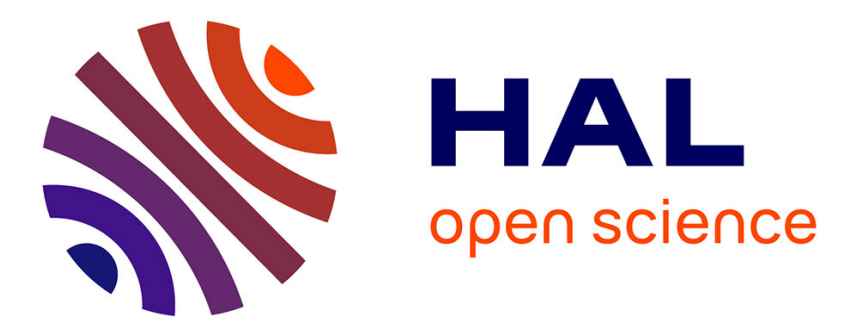

\title{
New hybrid algorithm based on nonmonotone spectral gradient and simultaneous perturbation
}

\author{
Zineb Tabbakh, Rachid Ellaia, Abderrahmane Habbal
}

\section{To cite this version:}

Zineb Tabbakh, Rachid Ellaia, Abderrahmane Habbal. New hybrid algorithm based on nonmonotone spectral gradient and simultaneous perturbation. International Journal of Mathematical Modelling and Numerical Optimisation, 2019, 9 (1), pp.1-23. 10.1504/IJMMNO.2019.096911 . hal-01944548

\author{
HAL Id: hal-01944548 \\ https://hal.inria.fr/hal-01944548
}

Submitted on 4 Dec 2018

HAL is a multi-disciplinary open access archive for the deposit and dissemination of scientific research documents, whether they are published or not. The documents may come from teaching and research institutions in France or abroad, or from public or private research centers.
L'archive ouverte pluridisciplinaire HAL, est destinée au dépôt et à la diffusion de documents scientifiques de niveau recherche, publiés ou non, émanant des établissements d'enseignement et de recherche français ou étrangers, des laboratoires publics ou privés. 


\title{
New Hybrid Algorithm based on Nonmonotone Spectral Gradient and Simultaneous Perturbation
}

\section{Tabbakh Zineb}

LERMA-EMI,

Mohammed V University in Rabat,

Mohammadia School of Engineers, BP. 765, Ibn Sina Avenue, Agdal,

Rabat, Morocco

E-mail: tabbakh.zineb@gmail.com

\section{Ellaia Rachid}

LERMA-EMI,

Mohammed V University in Rabat,

Mohammadia School of Engineers, BP. 765, Ibn Sina Avenue, Agdal,

Rabat, Morocco

E-mail: ellaia@emi.ac.ma

\section{Habbal Abderrahmane}

Université Côte d'Azur, Inria, CNRS, LJAD, UMR 7351, Parc Valrose, 06108 Nice, France.

E-mail: habbal@unice.fr

\begin{abstract}
In this paper, we introduce a new hybrid method called Nonmonotone Spectral Gradient and Simultaneous Perturbation (NSGSP). It combines the advantages of nonmonotone spectral gradient (NSG), and simultaneous perturbation (SP) methods. The main idea of our approach is to use the simultaneous perturbation (SP) method in order to get a non expensive estimate of the gradient, and exploit the good properties of the nonmonotone spectral gradient (NSG) method in order to compute an efficient line search. Several numerical experiments are provided. The results indicate that the new method is effective and outperforms most of other popular methods.
\end{abstract}

Keywords: Nonmonotone line search; spectral gradient method; Simultaneous Perturbation Stochastic Approximation

Biographical notes: Tabbakh Zineb is PhD student at Mohammed V University in Rabat, Mohammadia School of Engineers, Morocco.

Ellaia Rachid is full Professor at Mohammed V University in Rabat, Mohammadia School of Engineers, Morocco. He received his Doctoral Thesis from Toulouse University in Applied Mathematics and Optimization, and Ph.D. degree in 1992 in Applied and Computational Mathematics. He has held visiting professor positions at Toulouse university, Chile university, INSA of Rouen , Nice Sophia Antipolis University, Haute Alsace University and INRIA Lille. His interests are in Metaheuristics and Nature Inspired Computing, Optimization and Uncertainties, Finance, Global and Stochastic Optimization. Presently, he 
is President of the Moroccan Society of Nature-Inspired Computing and Metaheuristics (METAH).

Habbal Abderrahmane is Associate Professor at Nice Sophia-Antipolis University, and INRIA Sophia-Antipolis, France. Research activities of A. Habbal concern analysis and control of systems governed by partial differential equations (PDEs), optimization theory and algorithms and PDE-constrained games. Application fields are related to (nonlinear) mechanics, image processing, data recovering and cell dynamics.

\section{Introduction}

We consider minimization problems of the general form

$$
\begin{cases}\text { minimize } & f(x) \\ \text { subject to } & x \in S\end{cases}
$$

where $f$ is a smooth cost function and $S \subset \mathbf{R}^{n}$ defines the optimization constraints.

In the vast nonlinear optimization literature, many deterministic and stochastic methods have been developed to solve (1). Among the most popular are the steepest descent algorithms (It is out of the scope of the present paper to provide a review of these methods). From the latter family, spectral gradient methods, originated in Barzilai and Borwein (1988) have been applied successfully to find local minimizers of minimization problems for which computational issues arise such as cost and gradient evaluation is expensive, the Hessian is either not available or requires a prohibitive amount of storage. Notice that these methods are essentially steepest descent method, where an exact computation of the optimal step size along the direction of negative gradient is approximated by different effective choices, yielding as many various methods.

Barzilai and Borwein (1988) step size is computed from a two-point approximation to the secant equation required in quasi-Newton methods. Raydan (1997) presented the global Barzilai-Borwein (GBB) method based on the technique of nonmonotone line search, which was first introduced in Grippo et al. (1986). This technique allows the convergence to a minimum of a broader class of cost functions $f$, starting from any initial point $x_{0}$, than the usual well behaving case (strongly convex to say it roughly). Its main characteristic is the non-descent property.

Therefore, Birgin et al. (2000) have improved the work of Raydan (1997) and introduced the nonmonotone spectral projected gradient (NSPG) method. This approach was developed for the minimization of smooth functions on a closed convex set and has been successfully applied, see e.g. Lakhbab and Bernoussi (2016). On the other hand, simultaneous perturbation stochastic approximation method (SPSA) has been developed by Spall (2000) and used to solve the problem (1). In this method, the gradient is estimated using only two cost evaluations regardless of the dimension $n$ of the optimization space. In simultaneous perturbation stochastic approximation (SPSA), all component directions are perturbed simultaneously using perturbations which are vectors of independent random variables assumed to be symmetric, zero-mean, \pm 1 valued, and Bernoulli distributed.

Unlike standard finite difference approximations which require $2 n$ cost evaluations to estimate the gradient, Kiefer and Wolfowitz (1952), SPSA methods are very attractive as 
soon as the cost evaluation is computationally expensive. Indeed, it is the case for most engineering optimization problems, where cost functions are obtained through the solution of very large scale linear and nonlinear systems, and where closed or implementable forms of the gradient are generally not available.

The main objective of this paper is to propose a method to solve problem (1) combining the nonmonotone spectral gradient (NSG) with the simultaneous perturbation (SP) method. The descent direction is a nearly unbiased estime of the gradient provided by the simultaneous perturbation (SP) method. Along this direction, we use a spectral choice of the step, combined with a nonmonotone line search strategy allowing for the cost function to increase for a limited number of iterations. The advantages of the proposed algorithm are assessed on numerous benchmark functions and the results are compared with many of the existing methods. The obtained results illustrate the efficiency and robustness of our method to solve large scale problems for a broad class of smooth objective functions.

The remaining of the paper is organized in the following way. In Section 2, the new hybrid method is described. In Section 3, numerical results are presented for the solution of various benchmark problems. Finally, in Sect.4 the paper is concluded.

\section{NSG, SPSA and hybrid NSGSP methods}

We recall in this section the basic features of the Nonmonotone Spectral Gradient (NSG) and Simultaneous Perturbation (SP) methods ; then, we describe with more details our proposed Nonmonotone Spectral Gradient and Simultaneous Perturbation hybrid method (NSGSP), and finally shortly recall the penalization method which we use in (NSGSP) to deal with constrained optimization.

\subsection{Nonmonotone Spectral Gradient NSG method}

The Problem (1) is frequently numerically solved, seeking a local minimizer $\mathbf{x}^{*}$, by using iterative methods according to the following generic scheme

$$
\mathbf{x}_{k+1}=\mathbf{x}_{k}-\alpha_{k} g_{k}\left(\mathbf{x}_{k}\right)
$$

where $g_{k}$ is the gradient of $f$ at $\mathbf{x}_{k}$, and $\alpha_{k} \in \mathbf{R}^{+}$the step length, given initial $\mathbf{x}_{0} \in \mathbf{R}^{\mathbf{n}}$.

Instead of an exact and expensive computation of the optimal line search

$$
\alpha_{k}=\operatorname{argmin}_{\alpha} f\left(\mathbf{x}_{k}+\alpha g_{k}\left(\mathbf{x}_{k}\right)\right)
$$

the so-called spectral gradient methods aim at providing cheap yet effective approximations of the step length. By combining the nonmonotone line search of Grippo et al. (1986) with the spectral gradient choice of the step length of Barzilai and Borwein (1988), Raydan (1997) proposed the following choice

$$
\alpha_{k}=\frac{\mathbf{s}_{k-1}^{T} \mathbf{s}_{k-1}}{\mathbf{s}_{k-1}^{T} \mathbf{y}_{k-1}}
$$

where $\mathbf{s}_{k-1}=\mathbf{x}_{k}-\mathbf{x}_{k-1}$ and $\mathbf{y}_{k-1}=g_{k}-g_{k-1}$. 
This line search essentially enforces the following condition:

$$
f\left(\mathbf{x}_{k+1}\right) \leq \max _{0 \leq j \leq \min \{k, M\}} f\left(\mathbf{x}_{k-j}\right)+\gamma g_{k}^{T}\left(\mathbf{x}_{k+1}-\mathbf{x}_{k}\right),
$$

where $M$ is a nonnegative integer and $\gamma$ is a small positive number.

Among alternative approaches to compute a line search, let us mention the one based on safeguarded quadratic interpolation proposed in Dai et al. (2002). The following quadratic model is used to approximate the objective function at $f\left(\mathbf{x}_{k}+\lambda \mathbf{s}_{k-1}\right)$ :

$$
q_{k}(\lambda)=f_{k}+\lambda g_{k}^{T} \mathbf{s}_{k-1}+\frac{1}{2} \alpha_{k} \lambda^{2}\left\|\mathbf{s}_{k-1}\right\|^{2},
$$

where $\alpha_{k}=\frac{\mathbf{s}_{k-1}^{T} \mathbf{y}_{k-1}}{\mathbf{y}_{k-1}^{T} \mathbf{y}_{k-1}}$, and the above model satisfies two interpolation conditions

$$
\nabla q_{k}(0)=g_{k}^{T} \mathbf{s}_{k-1} \text { and } q_{k}(-1)=f_{k-1},
$$

which yields the following choice of stepsize formula

$$
\lambda_{k}=-\frac{\mathbf{s}_{k-1}^{T} \mathbf{s}_{k-1}}{2\left(f_{k-1}-f_{k}\right)+2 \mathbf{s}_{k-1}^{T} g_{k}} .
$$

After the initial choice of step size, the nonmonotone line search is used to determine the appropriate step length in the appropriate direction, based on the formula (6).

\subsection{Simultaneous perturbation}

In practice, exact values of the gradient of cost functions, notably those considered in engineering applications, are either not available or very costly to approximate by some finite difference scheme, due to the large number of variable and to expensive evaluation of the costs. In this case, nonmonotone spectral gradient (NSG) cannot be applied directly. To overcome the problem of the possibly prohibitive computational cost in gradient approximation, one may consider the simultaneous perturbation (SP) method due to Spall (2000).

The gradient estimate of $f($.$) when using simultaneous perturbation (SP) is given by$ the following centered formula

$$
\hat{g}_{k}\left(x_{k}\right)=\frac{f\left(x_{k}+c_{k} \Delta_{k}\right)-f\left(x_{k}-c_{k} \Delta_{k}\right)}{2 c_{k}}\left[\Delta_{k 1}^{-1}, \Delta_{k 2}^{-1}, \ldots, \Delta_{k n}^{-1}\right]^{T}
$$

where $c_{k}$ is a small positive number, and the components $\Delta_{k i}(i=1,2, \cdots, n)$ of the perturbation $\Delta_{k}=\left[\Delta_{k 1}, \Delta_{k 2}, \cdots, \Delta_{k n}\right]^{T}$ are independent, symmetrically distributed about zero with finite inverse and second moments, and satisfying $\left|\Delta_{k i}\right|<\alpha_{0}$, and $E\left(\left(\left|\Delta_{k i}^{-1}\right|\right)<\alpha_{1}\right.$ for all indices $(k, i)$ for some finite $\alpha_{1} 0$ and $\alpha_{1}$. A simple and popular choice for each component of $\Delta_{k}$ is a Bernoulli \pm 1 distribution with probability $\frac{1}{2}$.

As shown in Spall (1992), simultaneous perturbation (SP) provides an asymptotically unbiased estimate of the exact gradient $g_{k}$ while requiring only a fraction $\frac{1}{n}$ of the total cost spent in approximating the gradient with a centered finite difference scheme.

We recall hereafter the estimation theorem proved in Spall (1992), as well as a sketched proof (for it is simple enough, despite the striking claim of the theorem). 
Theorem 1: Let be $\Delta_{k}=\left[\Delta_{k 1}, \Delta_{k 2}, \cdots, \Delta_{k n}\right]^{T}$ that satisfy the above conditions, and suppose that $f$ is smooth enough, at least twice continuously differentiable, and noise-free. Then, $\hat{g}_{k}\left(x_{k}\right)$ is a nearly unbiased estimate of the $g_{k}\left(x_{k}\right)$ of $f$

$$
E\left(\hat{g}_{k}\left(x_{k}\right)\right)=g_{k}\left(x_{k}\right)+\mathcal{O}\left(c_{k}^{2}\right) \text { almost surely }
$$

where $g_{k}\left(x_{k}\right)$ is the true gradient of $f$ at the-deterministic-point $x_{k}$.

Proof 1: At some fixed iteration $k$, we take noise-free measurements of $f$ at $x_{k}+c_{k} \Delta_{k}$, using a standard second order Taylor series expansion,

$$
f\left(x_{k} \pm c_{k} \Delta_{k}\right)=f\left(x_{k}\right) \pm c_{k} g\left(x_{k}\right)^{T} \Delta_{k}+\frac{c_{k}^{2}}{2} \Delta_{k}^{T} \cdot \nabla^{2} f\left(x_{k}\right) \Delta_{k}+\mathcal{O}\left(c_{k}^{3}\left\|\Delta_{k}\right\|^{3}\right)
$$

Then, for $i=1 \cdots n$, one has

$$
E\left[\hat{g}_{k i}\left(x_{k}\right)\right]=E\left[\frac{f\left(x_{k}+c_{k} \Delta_{k}\right)-f\left(x_{k}-c_{k} \Delta_{k}\right)}{2 c_{k} \Delta_{k i}}\right]
$$

From (9) we have

$$
E\left[\hat{g}_{k i}\left(x_{k}\right)\right]=E\left[\frac{\left[c_{k} g\left(x_{k}\right)^{T} \Delta_{k}+\mathcal{O}\left(c_{k}^{3}\left\|\Delta_{k}\right\|^{3}\right)\right]-\left[-c_{k} g\left(x_{k}\right)^{T} \Delta_{k}+\mathcal{O}\left(c_{k}^{3}\left\|\Delta_{k}\right\|^{3}\right)\right]}{2 c_{k} \Delta_{k i}}\right]
$$

It follows that:

$$
\begin{aligned}
E\left[\hat{g}_{k i}\left(x_{k}\right)\right] & =E\left[\frac{2 c_{k} g\left(x_{k}\right)^{T} \Delta_{k}+\mathcal{O}\left(c_{k}^{3}\left\|\Delta_{k}\right\|^{3}\right)}{2 c_{k} \Delta_{k i}}\right] \\
& =E\left[\frac{2 c_{k} \sum_{j=1}^{n} g_{j}\left(x_{k}\right) \Delta_{k j}+\mathcal{O}\left(c_{k}^{3}\left\|\Delta_{k}\right\|^{3}\right)}{2 c_{k} \Delta_{k i}}\right] \\
& =g_{i}\left(x_{k}\right)+\sum_{j \neq i} g_{j}\left(x_{k}\right) E\left(\frac{\Delta_{k j}}{\Delta_{k i}}\right)+\mathcal{O}\left(c_{k}^{2} E\left[\left\|\Delta_{k}\right\|^{2}\right]\right) .
\end{aligned}
$$

Hence, under the conditions given above on $\Delta_{k}$,

$$
\forall j=1, \cdots, n E\left(\Delta_{k j}^{2}\right)=1, \text { and for all } j \neq i \quad E\left(\frac{\Delta_{k j}}{\Delta_{k i}}\right)=0 .
$$

Consequently,

$$
E\left[\hat{g}_{k i}\left(x_{k}\right)\right]=g_{i}\left(x_{k}\right)\left(x_{k}\right)+\mathcal{O}\left(c_{k}^{2}\right) .
$$

The bias of gradient estimation is of order $\mathcal{O}\left(c_{k}^{2}\right)$.

Remark 1: Notice that the proof above assumes that the point $x_{k}$ is deterministic, which no more holds when we shall consider iterates $x_{k}$ updated using the stochastic SP approximation. The proof however still holds in this case, by considering expectations conditional to $x_{k}$, see Spall (1992). 


\subsection{The proposed NSGSP method}

The nonmonotone spectral gradient and simultaneous perturbation (NSGSP) method combines the local search algorithm nonmonotone spectral gradient (NSG) with the descent method where the gradient is estimated by using the simultaneous perturbation. The recursive procedure (2) becomes:

$$
\hat{x}_{k+1}=\hat{x}_{k}-\alpha_{k} \hat{g}_{k}\left(\hat{x}_{k}\right),
$$

where $\hat{x}_{k}$ is the current point, $\hat{g}_{k}\left(\hat{x}_{k}\right)$ is the estimate gradient of $f$ at $\hat{x}_{k}$ obtained using simultaneous perturbation (SP), and $\alpha_{k}$ is the Barzilai-Borwein (BB) step size attained via nonmonotone line search.

\subsection{Handling the constraints in NSGSP}

To solve constrained optimization problems we have used a classical penalty method, owing to some encouraging properties such as the simplicity of these methods and their ease of implementation. These methods were originally proposed by Courant R. (1943) and developed among others by Carroll (1961) and Fiacco and McCormick (1966).

Applying penalty methods, we solve a sequence of unconstrained problems by adding to the objective function a penalty term for violation of constraints. The penalty function is weighted by an increasing sequence of large positive numbers, forcing the unconstrained solution to converge to a feasible one, that is, to satisfy all constraints. There are two types of methods: interior penalty methods, and exterior penalty methods. Since our problem has both inequality and equality constraints, we used exterior penalty functions and combined it with the NSGSP method to handle the constraints in the problem (1).

Assume that the feasible region $S$ of the minimization problem (1) is given by

$$
S=\left\{x: G_{i}(x) \leq 0, \quad i=1,2, \cdots, m \text { and } H_{j}(x)=0, \quad j=1,2, \cdots, p\right\} .
$$

where $m$ is the number of inequality constraints and $p$ the number of equality constraints. The problem (1) can be replaced by the unconstrained one as follows

$$
\min _{x \in \mathbf{R}^{n}} f(x)+\mu P(x),
$$

where $\mu$ is a positive parameter and $P$ is a penalty function of the form:

$$
P(x)=\sum_{i=1}^{m}\left(\max \left[0, G_{i}(x)\right]\right)^{2}+\sum_{j=1}^{p}\left|H_{j}(x)\right|^{2}
$$

$P$ is of course positive and continuous and $P(x)=0$ if and only if $x \in S$.

However, despite its implementation simplicity, the difficulty of the penalty function approach is to find an appropriate sequence of penalty parameters, that must be chosen large enough to converge to the solution of the constrained problem but not too large, to avoid degeneracy and ill conditioning. 


\section{Computational Experiments}

The proposed Nonmonotone Spectral Gradient and Simultaneous Perturbation (NSGSP) algorithm was implemented in Matlab. We have followed a Taguchi methodology to tune different parameters specific to the code, and then applied our method to a wide range of benchmark problems.

We assessed our algorithm versus a large number of unconstrained and constrained academic problems, and compared our obtained results to numerous popular methods. We thereafter led a comparative study on a worldwide referenced engineering optimization benchmarks : three bar truss, optimal control, pressure vessel design, tension-compression spring design and the speed reducer design.

For all the presented numerical experiments, the same stopping criteria were used : either the gradient norm was below some small tolerance threshold (relevant for unconstrained and penalized problems), or the maximum iterations were used without achieving convergence.

\subsection{Preliminary parameter design and analysis}

For the robust tuning of parameters specific to our code, we have used a Taguchi methodology. We have paid particular attention to three parameters : $\gamma, c$ and $M$. These parameters are not classical in descent methods, and a sensitivity analysis was then mandatory. After a preliminary analysis of the nonmonotone spectral gradient and simultaneous perturbation (NSGSP) algorithm, we have chosen three levels for each parameter, as described in Table 1. The aim is to estimate the parameters variation effects, in order to set up a robust choice of the latter.

The Taguchi method uses a loss function to measure the deviation between an experimental value and a desired one. See e.g. Peace (1993). This loss function is transformed into a signal-to-noise $(\mathrm{S} / \mathrm{N})$ ratio, so that a larger signal-to-noise $(\mathrm{S} / \mathrm{N})$ ratio corresponds to a better parameter design, that is, the parameter setting with the highest signal-to-noise $(\mathrm{S} / \mathrm{N})$ ratio $\mathrm{s}$ the best choice for the experiment. In the present study, the lower-the-better-quality characteristic in signal-to-noise ratio is considered

$$
\eta=-10 \log \frac{1}{m} \sum_{j=1}^{m} y_{j}^{2}
$$

where $y_{j}$ is the observed data in $j$ th experimentation and $m$ is the number of observations. The $L_{18}\left(3^{3}\right)$ orthogonal array (refer to Peace (1993) for details) shown in Table 2 was used to select the optimal parameters.

After the $\mathrm{S} / \mathrm{N}$ ratios are measured (Figure 1 and Table 2), the next step is to analyze their significance and effects based on analysis of variance (ANOVA). The results are shown in Table 3.

The results of the variance analysis of the parameters design experiment (Table 3 ) show that the parameter $c(\mathrm{~B})$ is the factor that represents the larger effect on the total variation in the function value, with a contribution amount of $37,91 \%$; the second factor is $\gamma$ with a contribution of $24,50 \%$. Then follows the effect of $M$ with a contribution of $14,62 \%$.

The Taguchi design experiment resulted in the choice of the following parameters for the nonmonotone spectral gradient and simultaneous perturbation (NSGSP) :

$\gamma=10^{-4}, \mathbf{M}=10$ and $\mathbf{c}=\mathbf{0 . 0 1}$. 
The simultaneous perturbation (SP) sequence is given by $c_{k}=\frac{c}{k^{\tau}}$ where $\tau=0.101$ and $k$ is the iteration counter.

The setup for other computational parameters is (see the significance in Algorithm 1) $\alpha_{\min }=10^{-30}, \alpha_{\max }=10^{30}, \sigma_{1}=0.1, \sigma_{2}=0.9$ and etol $=10^{-6}$.

Next, we use the following indicators : $S D$ for standard deviation, $S R$ for successful rate. The indicator $S R$ is used to calculate the number of successful runs, that is, when the algorithm generates a desired solution with a required accuracy. We have chosen an accuracy of $10^{-4}$ for all the tests we ran.

Furthermore, to illustrate the statistical significance of the performance of the proposed method, Cohen's $d$ statistical distance is used, for unconstrained optimization examples, to quantify the difference between two means. Each minimization example was run 50 times for twice, and Cohen's $d$ can then be calculated as:

$$
d=\frac{\text { Mean }_{1}-\text { Mean }_{2}}{\sigma_{\text {pooled }}} \quad \text { where } \quad \sigma_{\text {pooled }}=\sqrt{\frac{\left(S D_{1}^{2}+S D_{2}^{2}\right)}{2}}
$$

For our minimization benchmarks, the indicators $S D$ and $S R$, as well as the Cohen's $d$ statistics are reported in Table 7.

\subsection{Unconstrained optimization benchmark}

We consider a standard, widely used, benchmark list of unconstrained optimization problems. Objective functions, spaces and optima values are listed in Table 4 and in Table 5. These test case functions have different features making them challenging, like non convexity and/or multi-modality. They have known local and global minima.

The NSGSP algorithm introduced in the present paper was ran on these test cases, and the obtained results were compared to numerous other well proved algorithms listed in Table 6. The computational results and statistical metrics are presented in Table 7.

We have plotted in Figure 2 (Eason and Fenton function) and in Figure 3 (Aluffi-Pentini function) the evolution history of the objective functions with respect to the optimization iterations. These two profiles illustrate very clearly the non-descent property of the NSGSP algorithm (inherited from the NSG management of the iterates).

\subsection{Constrained optimization benchmark}

We have considered five constrained optimization test cases as detailed below. In Table 8 , we have reported the results obtained with the NSGSP method. These results are compared to the ones available from the literature.

\subsubsection{Constrained problem I:}

$$
\begin{aligned}
& f(x)=\left(x_{1}-2\right)^{2}+\left(x_{2}-1\right)^{2} \\
& \text { s.t, } \\
& g_{1}(x)=x_{1}-2 x_{2}+1=0 \\
& g_{2}(x)=x_{1}^{2} / 4-x_{2}^{2}+1 \geq 0 \\
& \quad-10 \leq x_{1}, x_{2} \leq 10 .
\end{aligned}
$$

The global minimum is attained at $x^{*}=(0.82288,0.91144)$, and $f\left(x^{*}\right)=1.3935$. 


\subsubsection{Constrained problem II:}

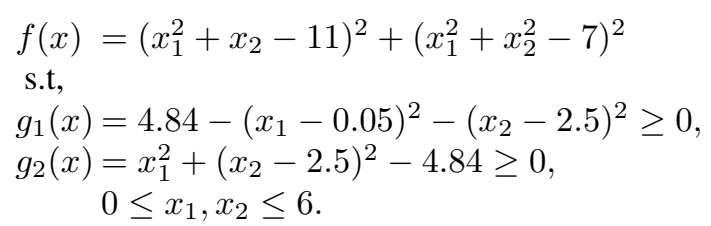

The global minimum is attained at $x^{*}=(2.246826,2.381865)$, and $f\left(x^{*}\right)=13.59085$, see Bracken and McCormick (1968).

\subsubsection{Constrained problem III:}

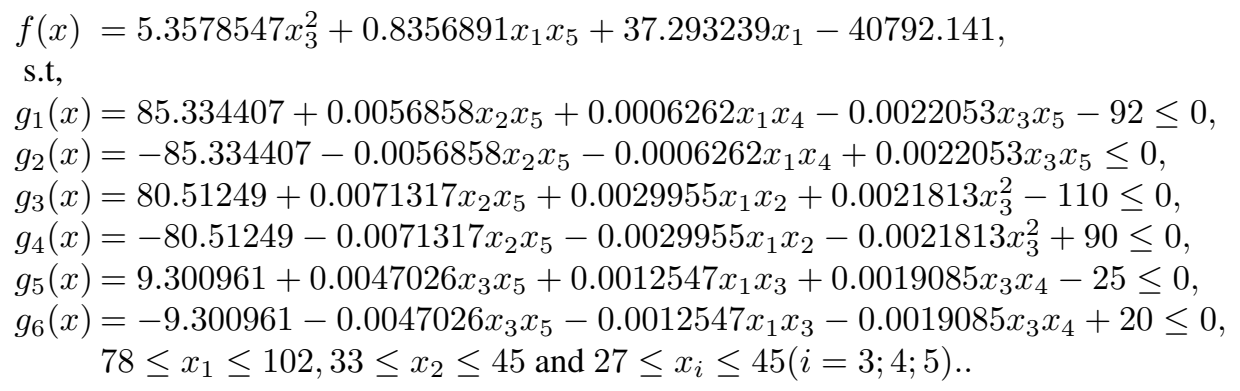

\subsubsection{Constrained problem IV}

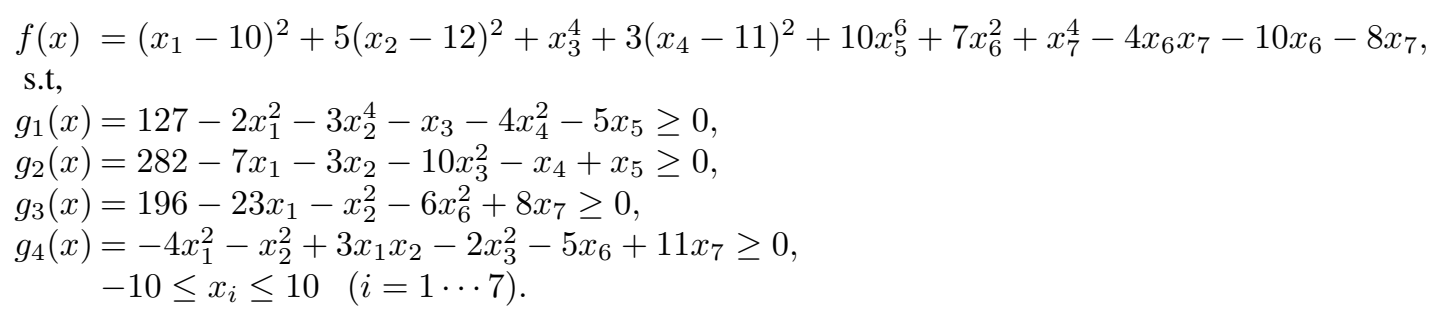

Optimal solution is $x^{*}=(2.330499,1.951372,-0.4775414,4.365726,-0.6244870,1.038131,1.594227)$, and $f\left(x^{*}\right)=680.6300573$. 


\subsubsection{Constrained problem $V$}

$$
\begin{aligned}
f(x) & =x_{1}^{2}+x_{2}^{2}+x_{1} x_{2}-14 x_{1}-16 x_{2}+\left(x_{3}-10\right)^{2}+4\left(x_{4}-5\right)^{2}+\left(x_{5}-3\right)^{2}+2\left(x_{6}-1\right)^{2}+5 x_{7}^{2} \\
& +7\left(x_{8}-11\right)^{2}+2\left(x_{9}-10\right)^{2}+\left(x_{10}-7\right)^{2}+45, \\
\text { s.t, } & \\
g_{1}(x) & =4 x_{1}+5 x_{2}-3 x_{7}+9 x_{8}-105 \geq 0, \\
g_{2}(x) & =10 x_{1}-8 x_{1}-17 x_{7}+2 x_{8} \geq 0, \\
g_{3}(x) & =-8 x_{1}+2 x_{2}+5 x_{9}-2 x_{10}-12 \geq 0, \\
g_{4}(x) & =3\left(x_{1}-2\right)^{2}+4\left(x_{2}-3\right)^{2}+2 x_{3}^{2}-7 x_{4}-120 \geq 0, \\
g_{5}(x) & =5 x_{1}^{2}+8 x_{2}+\left(x_{3}-6\right)^{2}-2 x_{4}-40 \geq 0, \\
g_{6}(x) & =x_{1}^{2}+2\left(x_{2}-2\right)^{2}-2 x_{1} x_{2}+14 x_{5}-6 x_{6} \geq 0, \\
g_{7}(x) & =0.5\left(x_{1}-8\right)^{2}+2\left(x_{2}-4\right)^{2}+3 x_{5}^{2}-x_{6}-30 \geq 0, \\
g_{8}(x) & =-3 x_{1}+6 x_{2}+12\left(x_{9}-8\right)^{2}-7 x_{10} \geq 0, \\
& -10 \leq x_{i} \leq 10 \quad(i=1 \cdots 10) .
\end{aligned}
$$

Optimal solution is $x^{*}=(2.1719,2.3636,8.7739,5.9598,0.9906,1.4305,1.3216,9.8287,8.2800,8.3759)$, and $f\left(x^{*}\right)=24.3062091$.

\subsection{Optimal Control problem}

The computational solution of optimal control problems is an important field in optimization theory. It has been studied by many workers, see e.g. Delprat et al. (2004), Delprat et al. (1996) and Mehra and Davis (1972).

We consider the following linear quadratic optimal control

$$
\left\{\begin{array}{c}
\min _{u} J\left(x_{0}, u\right)=\int_{0}^{\infty}\left[x(t)^{T} Q x(t)+u(t)^{T} R u(t)\right] d t \\
\text { subject to } \quad \dot{x}=A x+B u ; \quad x(0)=x_{0}
\end{array}\right.
$$

where $Q$ is a positive semidefinite matrix, $R$ is a positive definite matrix, and $x_{0}$ is given. The problem (25) can be solved by using the algebraic Riccati equation (see, for example Broucke (2012)), a powerful tool in linear control theory. Since the algebraic Riccati equation is a nonlinear algebraic equation, solving it analytically in dimensions greater than 3 is intractable in general.

Assumption 1: $(A, B)$ is stabilizable.

By stabilizability, there exists a feedback gain $K$ such that the closed loop system

$$
\dot{x}=(A-B K) x
$$

is stable. The feedback law $u=-K x$ is clearly admissible. The solution of 26 is given by

$$
x(t)=e^{(A-B K) t} x_{0}
$$

The cost criterion is then given by

$$
J\left(x_{0},-K x\right)=x_{0}^{T} \int_{0}^{\infty} \mathbf{e}^{(A-B K)^{T} t}\left(Q+K^{T} R K\right) \mathbf{e}^{(A-B K) t} d t x_{0}
$$

which is finite. Hence the optimization problem is meaningful. 


\subsubsection{An NSGSP illustration}

Consider the optimal control of a servomotor. Here $u(t)$ is a scalar control and $x \in \mathbf{R}^{2}$.

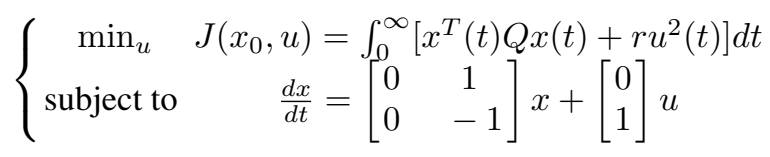

where $Q=\left[\begin{array}{ll}1 & 0 \\ 0 & 0\end{array}\right], r>0$.

We discretize problem (28) as follow

$$
\left\{\begin{array}{cc}
\text { minimize } & h\left(\sum_{1}^{n+1} y_{i}^{2}+r \sum_{1}^{n} u_{i}^{2}\right) \\
\text { subject to } & y_{i+1}-y_{i}-d t . z_{i}=0 \\
& z_{i+1}+(-1+d t) z_{i}-d t \cdot u_{i}=0
\end{array}\right.
$$

where $n=20$, and $x=(y, z)$; the integration step to approximate the cost function is $h=10^{-3}$ and the ODE time step is $d t=10^{-4}$.

We choose $r=0.5, u_{0}=-5 . \mathbb{1}_{n}$ and $x_{0}=[3.25 ; 4.95]$. By using nonmonotone spectral gradient and simultaneous perturbation (NSGSP) method in problem (29), we found $K \approx$ $[1.41 ; 0.96]$ and $J\left(x_{0}, u^{*}\right)=4.5712$. The optimal control is given by $u^{*}(t)=-1.41 y(t)-$ $0.96 z(t)$. The same result is obtained by using the Matlab command lqr.

\subsection{Pressure vessel design problem}

The pressure vessel design problem is a well known benchmark problem introduced in Kannan and Kramer (1994). The main objective is to minimise the cost $f(x)$ of the material mass, forming and welding. There are four design variables: $T s\left(=x_{1}\right)$ is the thickness of the shell, $T h\left(=x_{2}\right)$ is the spherical head thickness, $R\left(=x_{3}\right)$ is the inner radius, and $L\left(=x_{4}\right)$ is the length of the cylindrical section. Length unit is in inches.

In the literature, $T s$ and $T h$ are integer multiples of 0.0625 , and $x_{3}, x_{4}$ are continuous variables, such that $40 \leq x_{3} \leq 80$, and $20 \leq x_{4} \leq 60$.

The formulation of this problem can be written as follows:

$$
\left\{\begin{array}{c}
\text { minimize } f(x)=0.6224 x_{1} x_{3} x_{4}+1.7781 x_{2} x_{3}^{3}+3.1611 x_{1}^{2} x_{4}+19.84 x_{1}^{2} x_{3} \\
\text { subject to } g_{1}(x)=0.0193 x_{3}-x_{1} \geq 0 \\
g_{2}(x)=0.00954 x_{3}-x_{2} \geq 0 \\
g_{3}(x)=750.0 \times 1728.0-\pi x_{3}^{2} x_{4}-\frac{4}{3} \pi x_{3}^{2} \geq 0 \\
g_{4}(x)=x_{4}-240.0 \geq 0 \\
g_{5}(x)=1.1-x_{1} \geq 0 \\
g_{6}(x)=0.6-x_{2} \geq 0
\end{array}\right.
$$

The NSGSP method was applied to the pressure vessel design problem and the obtained results were compared to those of the literature (De KS Lee et al, Wu, Chow and Sandgren) as illustrated in Table 11. 


\subsection{Tension-compression spring design problem}

The tension/compression spring design problem is described in Arora (1989). The objective is to minimize the weight $f(x)$ of the tension compression spring (as shown in Figure 5 ). The optimization problem involves four nonlinear inequality constraints, namely the minimum deflection, shear stress, surge frequency, outside diameter, and bounds on the design variables. The design variables are wire diameter $d(=x 1)$, mean coil diameter $D(=$ $x 2$ ), and number of active coils $P(=x 3)$. The NSGSP obtained solution is given in Table 13 where it is compared to those obtained by other methods.

$$
\left\{\begin{aligned}
& \operatorname{minimize} f(x)=\left(x_{3}+2\right) x_{2} x_{1}^{2} \\
& \text { Subject to } g_{1}(x)=1-\frac{x_{2}^{2} x_{3}}{71785 x_{1}^{4}} \geq 0 \\
& g_{2}(x)=\frac{4 x_{2}^{2}-x_{1} x_{2}}{12566\left(x_{2} x_{1}^{3}-x_{1}^{4}\right)}+\frac{1}{5108 x_{1}^{2}}-1 \geq 0 \\
& g_{3}(x)=1-\frac{140.45 x_{1}}{x_{2}^{2} x_{3}} \geq 0 \\
& g_{4}(x)=\frac{x_{2}+x_{1}}{1.5}-1 \geq 0 \\
& 0.05 \leq x_{1} \leq 2.0,0.25 \leq x_{2} \leq 1.3,2 \leq x_{3} \leq 15
\end{aligned}\right.
$$

\subsection{Speed reducer design problem}

The speed reducer design problem is shown in Figure 6. The objective to minimize is the weight of the speed reducer subject to constraints on on bending stress of the gear teeth, surface stress, transverse deflections of the shafts, stresses in the shaft, and bounds on the design variables. The decision variables are the face width $b\left(=x_{1}\right)$, module of teeth $m\left(=x_{2}\right)$, number of teeth on pinion $z(=x 3)$, length of the first shaft between bearings $l_{1}\left(=x_{4}\right)$, length of the second shaft between bearings $l_{2}\left(=x_{5}\right)$, diameter of the first shaft $d_{1}\left(=x_{6}\right)$, and diameter of the second shaft $d_{2}\left(=x_{7}\right)$. 
The problem statement is the following :

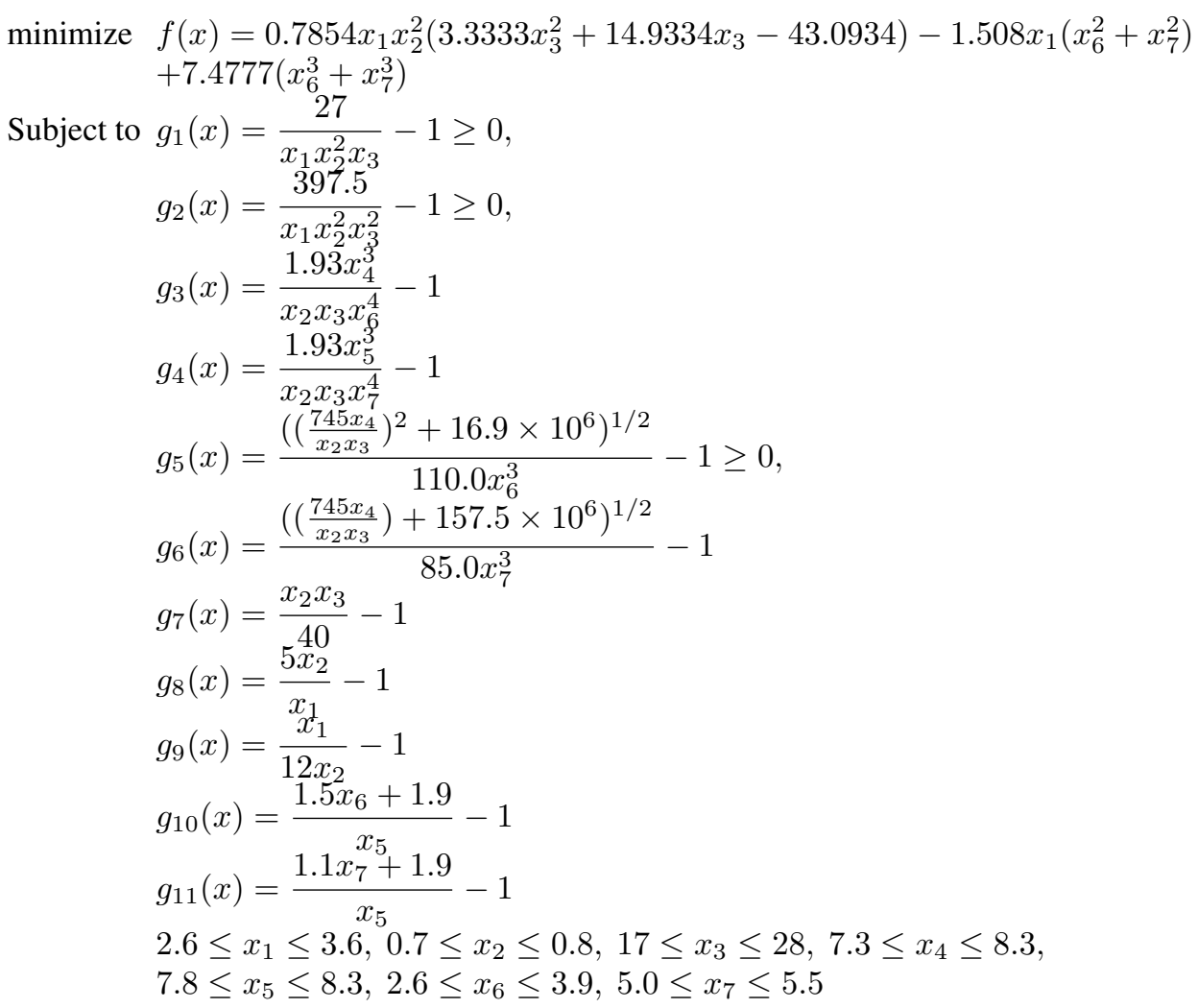

The results obtained by NSGSP are presented in Table 15, where they are compared to the results available in the literature.

\subsection{Three bar truss}

The three bar truss problem was introduced by Nowcki(1973). It is often used as a benchmark problem in structural optimization. The objective is to minimize the volume of a statically loaded 3-bar truss, subject to stress constraints on each member. The cross sectional areas $A_{i}$ are within the range $0.1<A_{i}<1$.

The mathematical programming formulation of this problem can be stated as follows:

$$
\left\{\begin{aligned}
\operatorname{minimize} & f(x)=\left(2 \sqrt{2} A_{1}+A_{2}\right) \times l \\
\text { subject to } g_{1}(x) & =\frac{\sqrt{2} A_{1}+A_{2}}{\sqrt{2} A_{1}^{2}+2 A_{1} A_{2}} P-\sigma \leq 0 \\
g_{2}(x) & =\frac{A_{2}}{\sqrt{2} A_{1}^{2}+2 A_{1} A_{2}} P-\sigma \leq 0 \\
g_{3}(x) & =\frac{1}{A_{1}+\sqrt{2} A_{2}} P-\sigma \leq 0 \\
0 & \leq A_{1} \leq 1,0 \leq A_{2} \leq 1
\end{aligned}\right.
$$

where $l=100 \mathrm{~cm}, P=2 \mathrm{KN} / \mathrm{cm}^{2}$ and $\sigma=2 \mathrm{KN} / \mathrm{cm}^{2}$.

The NSGSP results are presented in Table 16, and compared to other methods in Table 17. 


\section{Conclusion}

The present paper introduces a new minimization method called the nonmonotone spectral gradient and simultaneous perturbation (NSGSP). This method is applicable when the gradient of the objective function is not available or is expensive to compute. The gradient is replaced by a simultaneous perturbation stochastic approximation (SPSA). It then applies the Barzilai-Borwein step size and uses the nonmonotone line search techniques, which greatly improves the efficiency of the method. with the non monotone line search, the algorithm was able to escape the local minimum to better one. In many test cases, the new hybrid method exhibited some nice global optimization abilities.

The proposed method was applied to well-known benchmark functions and different classical problems, including twenty unconstrained, five constrained minimization problems, and structural optimization problems. All obtained results contribute to demonstrate the effectiveness of our method. The results were compared to those of published works found in literature. In all examples, nonmonotone spectral gradient and simultaneous perturbation (NSGSP) gave very satisfactory results, which indicates that the nonmonotone spectral gradient and simultaneous perturbation (NSGSP) is competitive, and can be used as an optimization technique for solving engineering optimization problems in challenging (non convex, multimodal) problems. A next step is to adapt the NSGSP approach in order to tackle multiobjective optimization problems, the aim being to capture the Pareto Frontier for large scale problems (those known as many-objective problems).

\section{References}

B. Raphael and I.F.C. Smith.(2003) 'A direct stochastic algorithm for global search.' Applied Mathematics and Computation, 146:729-758.

C. Zhang, J. Ning, S. Lu, D. Ouyang, and T. Ding. (2009) 'A novel hybrid differential evolution and particle swarm optimization algorithm for unconstrained optimization',Operations Research Letters, 37:117-122.

E.G. Birgin, J.M. Martínez, and M. Raydan.(2000) 'Nonmonotone spectral projected gradient methods on convex sets.' SIAM Journal on Optimization, 10:1196-1211.

I.G. Tsoulos.(2008) 'Modifications of real code genetic algorithm for global optimization.'Applied Mathematics and Computation, 203:598-607

Jerome Bracken and Garth P McCormick.(1968) 'Selected applications of nonlinear programming.' Technical report, DTIC Document.

J. Barzilai and J. M. Borwein.(1988) 'Two point step size gradient methods.' IMA Journal of Numerical Analysis, 8:141-148.

J. Kiefer and J. Wolfowitz.(1952) 'Stochastic estimation of the maximum of a regression function.' Ann. Statist, 23:462-466.

James C. Spall.(2000) 'Adaptive stochastic approximation by the simultaneous perturbation method.' IEEE Transactions on Automatic Control, 45:1839-1853. 
Halima Lakhbab and Souad El Bernoussi.(2016) 'Hybrid nonmonotone spectral gradient method for the unconstrained minimization problem.'Computational and Applied Mathematics, pages 1-10.

K. Deep and M. Thakur. (2007) 'A new mutation operator for real coded genetic algorithms.' Applied Mathematics and Computation, 193:211-230.

L. Wei and M. Zhao.(2005)'A niche hybrid genetic algorithm for global optimization of continuous multimodal functions.'Applied Mathematics and Computation, 160:649-661.

L. Grippo, F. Lampariello, and S. Lucidi.(1986)'A nonmonotone line search technique for newton's method.' SIAM Journal on Numerical Analysis, 23:707-716.

M. Broucke.(2012)'Linear geometric control theory.' Courses Notes, Toronto University, pages $141-147$.

M. Raydan.(1997) 'The barzilai and borwein gradient method for the large scale unconstrained minimization problem.' SIAM Journal on Optimization, 7:26-33.

M. Duran Toksary.(2006)'A heuristic approach to find the global optimum of function.' Journal of Computational and Applied Mathematics, 209:160-166.

Q. Yuan, Z. He, and H. Leng. (2008) 'A hybrid genetic algorithm for a class of global optimization problems with box constraints.' Applied Mathematics and Computation, 197:924-929.

R.K. Mehra and R Davis.(1972) 'A generalized gradient method for optimal control problems with inequality constraints and singular arcs.' IEEE Transactions on Automatic Control, AC-17:69-79.

S. Karmakar, S.K. Mahato, and A.K. Bhunia.(2009) 'Interval oriented multi-section techniques for global optimization.' Journal of Computational and Applied Mathematics, 224:3476-491.

S. Delprat, J. Lauber, T.M. Guerra, and J. Rimaux.(1996) 'The gradient projection method for solving an optimal control problem.'Applicationes Mathematica, 24:141-147.

S. Delprat, J. Lauber, T.M. Guerra, and J. Rimaux.(2004) 'Control of a parallel hybrid powertrain: Optimal control.' IEEE Transactions on Vehicular Technology, 53:872-881.

Yi-Tung Kao and Erwie Zahara.(2008) 'A hybrid genetic algorithm and particle swarm optimization for multimodal functions.'Appl. Soft Comput., 8:849-857.

Y.D. Kwon, S.B. Kwon, Seung-Bo J., and J.Y. Kim.(2003) 'Convergence enhanced genetic algorithm with successive zooming method for solving continuous optimization problems.' Comput. Struct., 81:1715-1725.

Y. Dai, J. Yuan, and Y.X. Yuan.(2002) 'Modified two-point stepsize gradient method for unconstrained optimization.' Comput. Optim. Appl, 20:103-109.

Z. Xinchao.(2010) 'A perturbed particle swarm algorithm for numerical optimization.' Applied Soft Computing, 10:119-124. 
Spall, J. C. (1992). Multivariate stochastic approximation using a simultaneous perturbation gradient approximation.IEEE transactions on automatic control, 37(3), 332-341.

Courant, R. (1943). Variational methods for the solution of problems of equilibrium and vibrations. Bulletin of the American mathematical Society, 49(1), 1-23.

Fiacco, A. V., \& McCormick, G. P. (1966). Extensions of SUMT for nonlinear programming: equality constraints and extrapolation. Management Science, 12(11), 816-828.

Carroll, C. W. (1961). The created response surface technique for optimizing nonlinear, restrained systems. Operations research, 9(2), 169-184.

Peace, G. S. (1993). Taguchi methods: a hands-on approach. Addison Wesley Publishing Company.

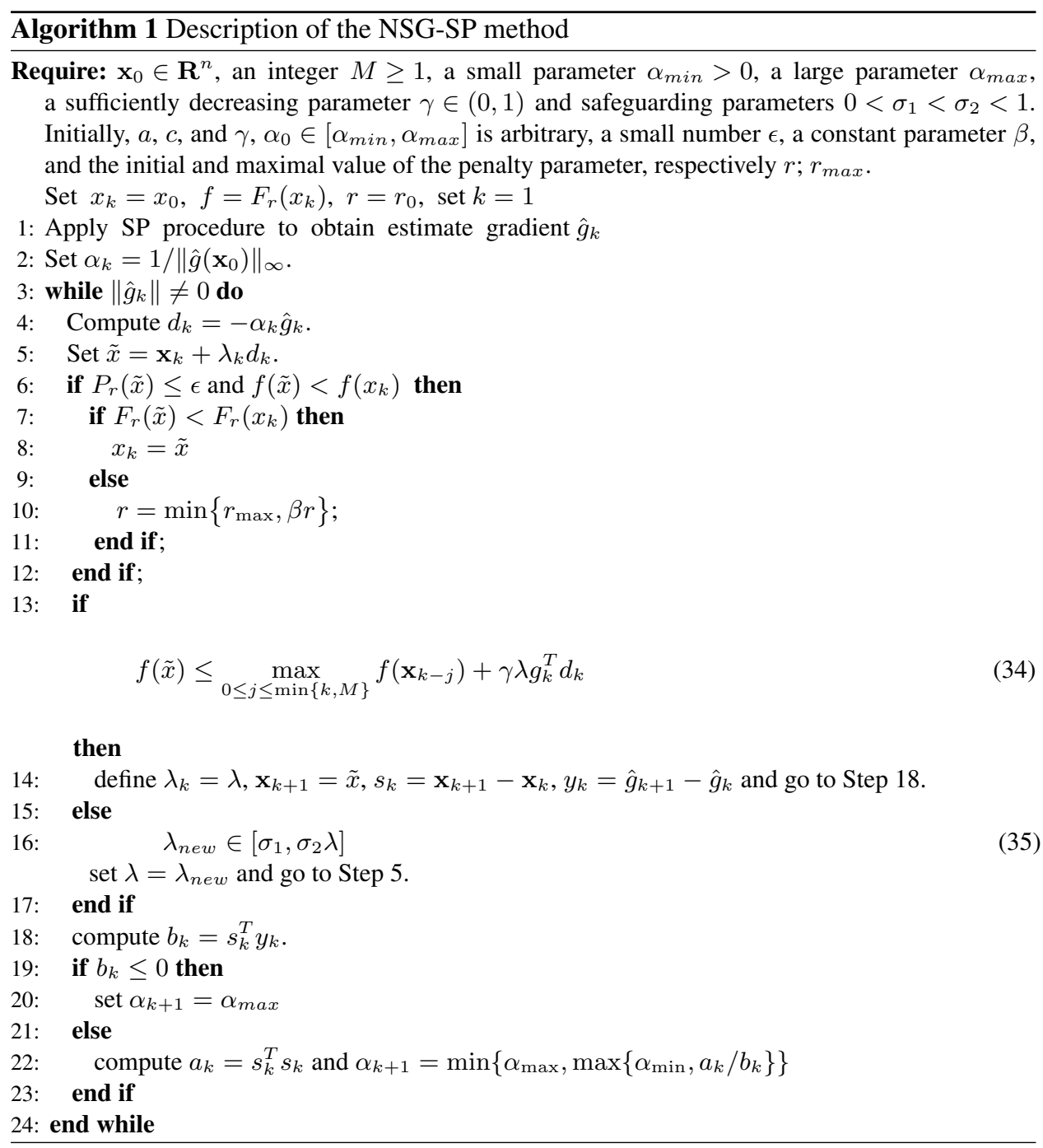




\begin{tabular}{llll}
\hline Parameters/Level & 1 & 2 & 3 \\
\hline$M$ & 5 & 10 & 20 \\
$c$ & 0.0001 & 0.01 & 1 \\
$\gamma$ & 0.0001 & 0.01 & 1 \\
\hline
\end{tabular}

Table 1 NSGSP parameters and their levels

\begin{tabular}{lllll}
\hline$M$ & $c$ & $\gamma$ & value of $f(x)$ & S/N ratio \\
\hline 5 & 0,0001 & 0,0001 & 0,3979 & 8,00452 \\
5 & 0,0100 & 0,0100 & 0,3979 & 8,00452 \\
5 & 1,0000 & 1,0000 & 0,6016 & 4,41384 \\
10 & 0,0001 & 0,0100 & 0,3979 & 8,00452 \\
10 & 0,0100 & 1,0000 & 0,4386 & 7,15863 \\
10 & 1,0000 & 0,0001 & 0,4679 & 6,59694 \\
20 & 0,0001 & 1,0000 & 0,3979 & 8,00452 \\
20 & 0,0100 & 0,0001 & 0,3979 & 8,00452 \\
20 & 1,0000 & 0,0100 & 0,4031 & 7,89174 \\
\hline
\end{tabular}

Table 2 Tuning NSGSP parameters : S/N ratios values of experiments.

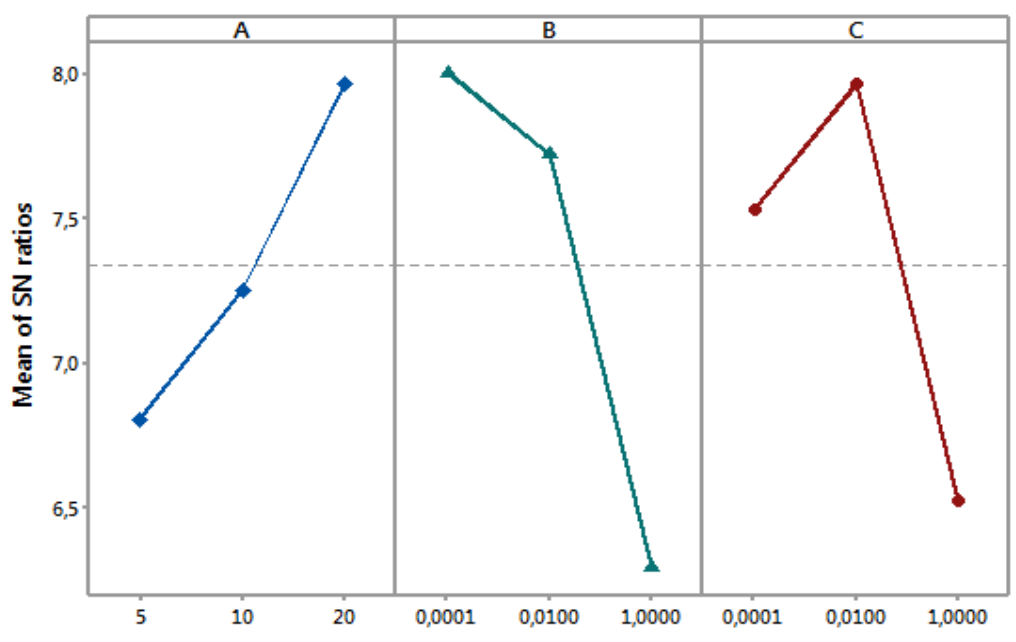

Figure 1: Main Effects Plot for SN ratios for the $(M, c, \gamma)$ NSGSP parameters

\begin{tabular}{lllllll}
\hline $\begin{array}{l}\text { Variance } \\
\text { source }\end{array}$ & $\begin{array}{l}\text { Degree of } \\
\text { freedom } \\
\text { (DF) }\end{array}$ & $\begin{array}{l}\text { adjusted } \\
\text { sum } \\
\text { square }\end{array}$ & $\begin{array}{l}\text { adjusted } \\
\text { mean } \\
\text { square }\end{array}$ & F-value & P-value & $\begin{array}{l}\text { Contribution } \\
\text { rate }(\%)\end{array}$ \\
\hline$M$ & 2 & 0,006576 & 0,003288 & 1,36 & 0,423 & 14,62 \\
$c$ & 2 & 0,015131 & 0,007566 & 3,14 & 0,241 & 37,91 \\
$\gamma$ & 2 & 0,010203 & 0,005102 & 2,12 & 0,321 & 24,50 \\
Error & 2 & 0,004817 & 0,002409 & & & \\
Total & 8 & 0,036728 & & & & \\
\hline
\end{tabular}

Table 3 Results of ANOVA : analysis of variance on the tuned parameters. 
Table 4 Test functions

\begin{tabular}{|c|c|c|}
\hline Test/Benchmark functions & Search region & Optimal value \\
\hline \multicolumn{3}{|l|}{ Griewank's function } \\
\hline$f_{1}=1+\sum_{i=1}^{N} \frac{x_{i}^{2}}{4000}-\prod_{i=1}^{N}\left(\cos \left(x_{i} / \sqrt{i}\right)\right)$ & $x \in \mathbf{R}^{N}$ & 0 \\
\hline \multicolumn{3}{|l|}{ Rastrigin's function } \\
\hline$f_{2}=10 N+\sum_{i=1}^{N}\left[x_{i}^{2}-10 \cos \left(2 \pi x_{i}\right)\right]$ & $x \in[-5,12 ; 5,12]^{N}$ & 0 \\
\hline \multicolumn{3}{|l|}{ Alluffi-Pentiny's function } \\
\hline$f_{3}=\frac{1}{4} x_{1}^{4}-\frac{1}{2} x_{1}^{2}+\frac{1}{10} x_{1}+\frac{1}{2} x_{2}^{2}$ & $x \in[-10 ; 10]^{2}$ & -0.352386 \\
\hline \multicolumn{3}{|l|}{ Bohachevsky's function } \\
\hline$f_{4}=x_{1}^{2}+2 x_{2}^{2}-\frac{3}{10} \cos \left(3 \pi x_{1}\right)-\frac{4}{10} \cos \left(4 \pi x_{2}\right)+\frac{7}{10}$ & $x \in[-100 ; 100]^{2}$ & 0 \\
\hline \multicolumn{3}{|l|}{ Branin function } \\
\hline$f_{5}=\left(x_{2}-\frac{5.1}{4 \pi} x_{1}^{2}-\frac{5}{\pi} x_{1}-6\right)+10\left(1-\frac{1}{8 \pi}\right) \cos \left(x_{1}\right)+10$ & $x_{1} \in[-5 ; 10], x_{2} \in[0 ; 15]$ & 0.397887 \\
\hline \multicolumn{3}{|l|}{ Camel's function } \\
\hline$f_{6}=4 x_{1}^{2}-2.1 x_{1}^{4}+\frac{1}{3} x_{1}^{5}+x_{1} x_{2}-4 x_{2}^{2}+4 x_{2}^{4}$ & $x \in[-5 ; 5]^{2}$ & -1.0316 \\
\hline \multicolumn{3}{|l|}{ Shubert function } \\
\hline $\begin{array}{l}f_{9}=-\sum_{i=1}^{2} \sum_{j=1}^{5} j \sin \left(x_{i}(j+1)\right)+1 \\
\text { Pen Holder Function }\end{array}$ & $x \in[-10 ; 10]^{2}$ & -24.06249 \\
\hline$f_{10}=-\exp \left\{-\left|\cos \left(x_{1}\right) \cos \left(x_{2}\right) e^{\left|1-\left[\left(x_{1}^{2}+x_{2}^{2}\right)^{0.5} / \pi\right]\right|}\right|^{-1}\right\}$ & $x \in[-11 ; 11]^{2}$ & -0.963540 \\
\hline $\begin{array}{l}f_{11}=\sum_{i=1}^{n}\left(x_{i}^{4}-16 x_{i}^{2}+5 x_{i}\right) \\
\text { Cosine Mixture Function }\end{array}$ & $x \in[-4 ; 4]^{n}$ & -78.33236 \\
\hline $\begin{array}{l}f_{12}=\sum_{i=1}^{n} x_{i}^{2}-0.1 \sum_{i=1}^{n} \cos \left(5 \pi x_{i}\right) \\
\text { Rosenbrock Function }\end{array}$ & $x \in[-1 ; 1]^{n}$ & $-0.1 \mathrm{n}$ \\
\hline$f_{13}=x_{1}^{2}+2 x_{2}^{2}-0.3 \cos \left(3 \pi x_{1}\right)-0.4 \cos \left(4 \pi x_{2}\right)+0.7$ & $x \in \mathbf{R}^{2}$ & 0 \\
\hline \multicolumn{3}{|l|}{ Bird Function } \\
\hline$f_{16}=\sin \left(x_{1}\right) \exp \left[\left(1-\cos \left(x_{2}\right)\right)^{2}\right]+\cos \left(x_{2}\right) \exp \left[\left(1-\sin \left(x_{1}\right)\right)^{2}\right]+\left(x_{1}-x_{2}\right)^{2}$ & $x \in[-2 \pi, 2 \pi]^{2}$ & -106.764537 \\
\hline \multicolumn{3}{|l|}{ Sphere Function } \\
\hline Zakharov Function & $x \in[-5.12,5.12]^{n}$ & 0 \\
\hline $\begin{array}{l}f_{18}=\sum_{i=1}^{n} x_{i}^{2}+\left(\sum_{i=1}^{n} 0.5 i x_{i}\right)^{2}+\left(\sum_{i=1}^{n} 0.5 i x_{i}\right)^{4} \\
\text { Six Hump Camel Back Function }\end{array}$ & $x \in[-100,100]^{n}$ & 0 \\
\hline$f_{19}=\left(4-2.1 x_{1}^{2}+\frac{x_{1}^{4}}{3}\right) x_{1}^{2}+x_{1} x_{2}+\left(-4+4 x_{2}^{2}\right) x_{2}^{2}$ & $x_{1} \in[-3,3], x_{2} \in[-2,2]$ & -1.031600 \\
\hline \multicolumn{3}{|l|}{ Exponential function } \\
\hline$f_{20}=\exp \left(0.5 \sum_{i=1}^{n} x_{i}^{2}\right)$ & $x \in[-1,1]^{n}$ & 1.00000 \\
\hline $\begin{array}{l}\text { Eason and Fenton Function } \\
f_{21}=\frac{1}{10}\left(12+x_{1}^{2}+\frac{1+x_{2}^{2}}{x_{1}^{2}}+\frac{x_{1}^{2} x_{2}^{2}+100}{\left(x_{1} x_{2}\right)^{4}}\right)\end{array}$ & $x \in[0,10]^{2}$ & 1.74 \\
\hline
\end{tabular}


Table 5 Test functions

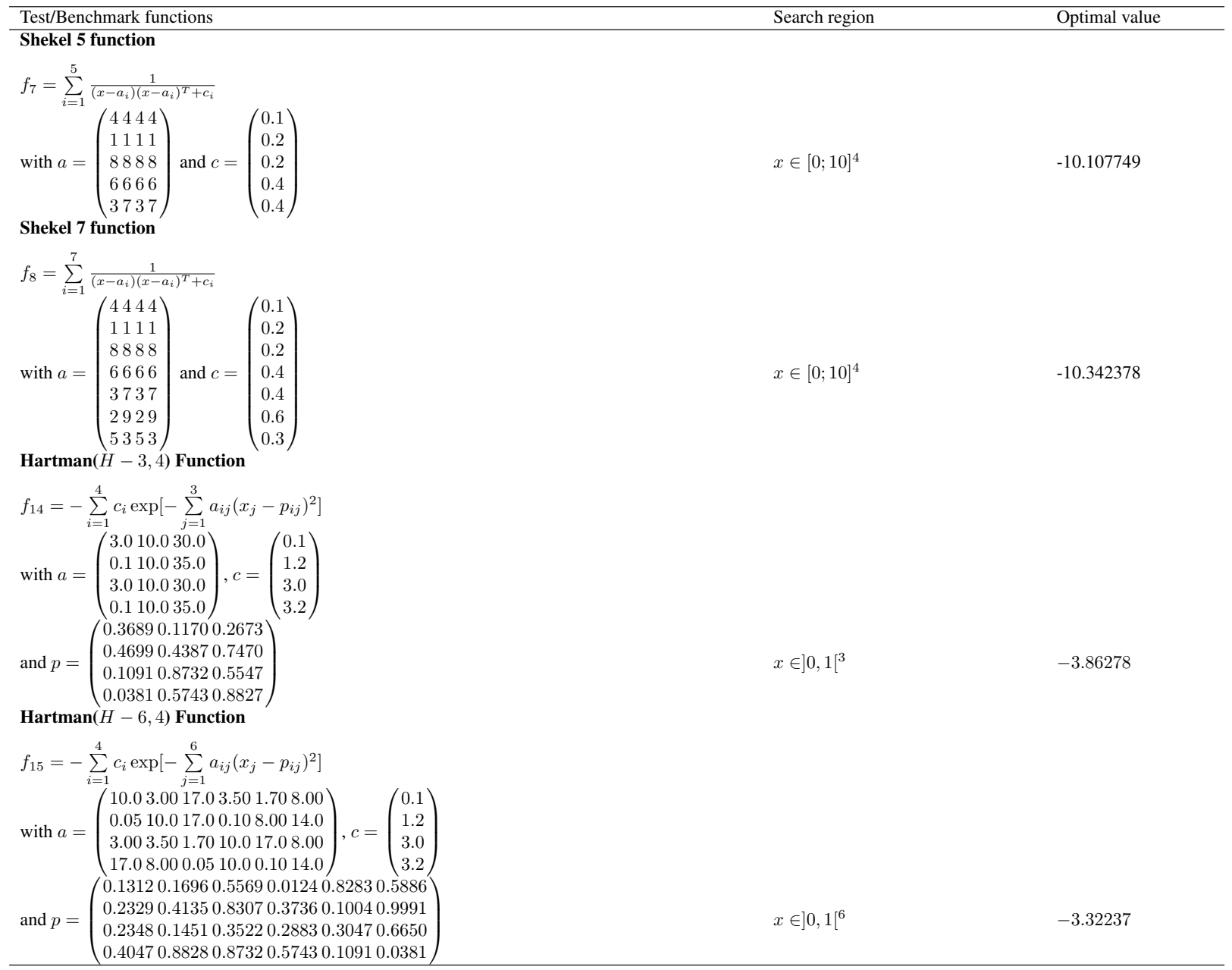

Table 6 Optimization methods used for performance analysis

\begin{tabular}{lll}
\hline Method & Name (Reference) & Used test function \\
\hline PGSL & Probabilistic global search lausanneRaphael and Smith (2003) & $f_{1}$ \\
DE-PSO & Differential Evolution-Particle Swarm OptimizationZhang et al. (2009) & $f_{18}$ \\
NHGA & Niche Hybrid Genetic AlgorithmWei and Zhao (2005) & $f_{18}$ \\
ESGAT & simple genetic algorithmRaphael and Smith (2003) & $f_{1}$ \\
Genitor & steady state genetic algorithmRaphael and Smith (2003) & $f_{1}$ \\
pPSA & perturbed Particle Swarm AlgorithmXinchao (2010) & $f_{17}$ \\
GAT & The Genetic Algorithm Toolbox in MATLABYuan et al. (2008) & $f_{2}$ \\
HGA & Hybrid Genetic AlgorithmYuan et al. (2008) & $f_{2}$ \\
MGA & Modified Genetic AlgorithmTsoulos (2008) & $f_{3}, f_{4}, f_{5}, f_{6}, f_{7}, f_{8}, f_{9}$ \\
MACO & The modified ant colony optimizationToksary (2006) & $f_{13}$ \\
SZGA & Successive zooming genetic algorithmKwon et al. (2003) & $f_{13}$ \\
LX-PM & Laplace crossover with Power MutationDeep and Thakur (2007) & $f_{17}$ \\
CTSS & Continuous Tabu Simplex Search & $f_{18}$ \\
GA-PSO & Genetic algorithms and particle swarm optimizationKao and Zahara (2008) & $f_{14}, f_{15}$ \\
\hline
\end{tabular}


Table 7 Computational results of test functions

\begin{tabular}{|c|c|c|c|c|c|c|c|c|c|c|}
\hline \multirow[t]{2}{*}{ Problem no. } & \multicolumn{8}{|c|}{ NSGSP method } & \multicolumn{2}{|c|}{ Other Methods } \\
\hline & Dimension & The Best & The Worst & Mean & SD & Median & SR & Cohen d & Method & Obtained result \\
\hline \multirow[t]{9}{*}{1} & 10 & 0 & $2.2204 \mathrm{e}-15$ & $6.8834 \mathrm{e}-17$ & $9.7074 \mathrm{e}-32$ & 0 & $100 \%$ & 0.14584 & ESGAT & 0.0515 \\
\hline & & & & & & & & & Genitor & 0.00496 \\
\hline & & & & & & & & & PGSL & 0.0007 \\
\hline & 20 & 0 & $4.4409 \mathrm{e}-16$ & $9.1038 \mathrm{e}-17$ & $1.3652 \mathrm{e}-32$ & 0 & $100 \%$ & 0.057556 & ESGAT & 0.0622 \\
\hline & & & & & & & & & Genitor & 0.0240 \\
\hline & & & & & & & & & PGSL & 0.0002 \\
\hline & 50 & $1.1102 \mathrm{e}-16$ & $1.8874 \mathrm{e}-15$ & $5.9286 \mathrm{e}-16$ & $1.5314 \mathrm{e}-31$ & $4.4409 \mathrm{e}-16$ & $100 \%$ & 0 & ESGAT & 0.0990 \\
\hline & & & & & & & & & Genitor & 0.0170 \\
\hline & & & & & & & & & PGSL & 0.0 \\
\hline \multirow[t]{5}{*}{2} & 10 & 0 & $2.8422 \mathrm{e}-14$ & $5.6843 \mathrm{e}-16$ & $1.5833 \mathrm{e}-29$ & 0 & $100 \%$ & 0 & HGA & $1.29 \mathrm{e}-12$ \\
\hline & & & & & & & & & GAT & 1.37 \\
\hline & 20 & 0 & $2.8422 \mathrm{e}-14$ & $3.4106 \mathrm{e}-15$ & $8.5303 \mathrm{e}-29$ & 0 & $100 \%$ & 0.29515 & HGA & $3.5 \mathrm{e}-12$ \\
\hline & & & & & & & & & GAT & 20.65 \\
\hline & 50 & 0 & $5.6843 \mathrm{e}-13$ & $1.2164 \mathrm{e}-13$ & $1.5123 \mathrm{e}-26$ & $1.1369 \mathrm{e}-13$ & $100 \%$ & -0.10723 & HGA & $9.09 \mathrm{e}-12$ \\
\hline 3 & 2 & -0.35239 & -0.35239 & -0.35239 & $1.4828 \mathrm{e}-31$ & -0.35239 & $100 \%$ & 0 & MGA & -0.352386 \\
\hline 4 & 2 & 0 & $2.2204 \mathrm{e}-16$ & $8.8818 \mathrm{e}-18$ & $1.8933 \mathrm{e}-33$ & 0 & $100 \%$ & 0.28577 & MGA & 0 \\
\hline 5 & 2 & 0.39789 & 0.39789 & 0.39789 & $3.469 \mathrm{e}-21$ & 0.39789 & $100 \%$ & 0.2092 & MGA & 0.397887 \\
\hline 6 & 2 & -1.0316 & -1.0316 & -1.0316 & $3.7964 \mathrm{e}-31$ & -1.0316 & $100 \%$ & 0 & MGA & -1.0316 \\
\hline 7 & 4 & -10.1532 & -10.1532 & -10.1532 & $3.96 \mathrm{e}-24$ & -10.1532 & $100 \%$ & 0.1009 & MGA & -10.107749 \\
\hline 8 & 4 & -10.4029 & -10.4029 & -10.4029 & $2.743 e-24$ & -10.4029 & $100 \%$ & 0.0118 & MGA & -10.342378 \\
\hline 9 & 2 & -186.7309 & -186.7309 & -186.7309 & $1.5304 \mathrm{e}-13$ & -186.73 & $100 \%$ & 0.245 & MGA & -24.06249 \\
\hline 10 & 2 & -1 & -0.99998 & -1 & $3.6028 \mathrm{e}-11$ & -1 & $100 \%$ & -0.13288 & Karmakar et al. (2009) & -0.963540 \\
\hline 11 & 2 & -78.3323 & -78.332 & -78.332 & $4.2005 \mathrm{e}-28$ & -78.332 & $100 \%$ & 0 & Karmakar et al. (2009) & -78.3323 \\
\hline 12 & 6 & -0.6 & -0.6 & -0.6 & $4.3289 \mathrm{e}-31$ & -0.6 & $100 \%$ & 0 & Karmakar et al. (2009) & -0.2 \\
\hline 13 & 2 & $3.6778 \mathrm{e}-16$ & $3.3111 \mathrm{e}-11$ & $9.3907 \mathrm{e}-13$ & $2.403 \mathrm{e}-23$ & $4.8213 \mathrm{e}-16$ & $100 \%$ & 0.19809 & SZGA & $2.98 \mathrm{e}-8$ \\
\hline & & & & & & & & MACO & 0 & \\
\hline 14 & 3 & -3.8634 & -3.8634 & -3.8634 & $4.5517 \mathrm{e}-30$ & -3.8634 & $100 \%$ & 0 & GA-PSO & -3.86278 \\
\hline 15 & 6 & -3.3224 & -3.3224 & -3.3224 & $9.4663 \mathrm{e}-32$ & -3.3224 & $100 \%$ & 0 & GA-PSO & -3.32237 \\
\hline 16 & 2 & -106.764 & -106.76 & -106.76 & $6.7855 \mathrm{e}-28$ & -106.76 & & & (Karmakar et al. (2009)) & -106.764537 \\
\hline 17 & 30 & $6.035 \mathrm{e}-31$ & $2.35 \mathrm{e}-26$ & $1.08 \mathrm{e}-27$ & $1.239 \mathrm{e}-53$ & $5.493 \mathrm{e}-29$ & $100 \%$ & 0.1186 & pPSA & $5.6 \mathrm{e}-06$ \\
\hline & & & & & & & & & LX-PM & $4.75 \mathrm{e}-11$ \\
\hline 18 & 30 & $6.9125 \mathrm{e}-34$ & $1.0671 \mathrm{e}-28$ & $4.2324 \mathrm{e}-30$ & $2.788 \mathrm{e}-58$ & $7.9539 \mathrm{e}-32$ & $100 \%$ & -0.17629 & CTSS & $3 e-07$ \\
\hline 19 & 2 & -1.0316 & -1.0316 & -1.0316 & $4.1908 \mathrm{e}-31$ & -1.0316 & $100 \%$ & -0.39693 & (Karmakar et al. (2009)) & -1.031628 \\
\hline 20 & 10 & 1 & 1 & 1 & $4.1612 \mathrm{e}-31$ & 1 & $100 \%$ & 0 & (Karmakar et al. (2009)) & 1.00000 \\
\hline
\end{tabular}

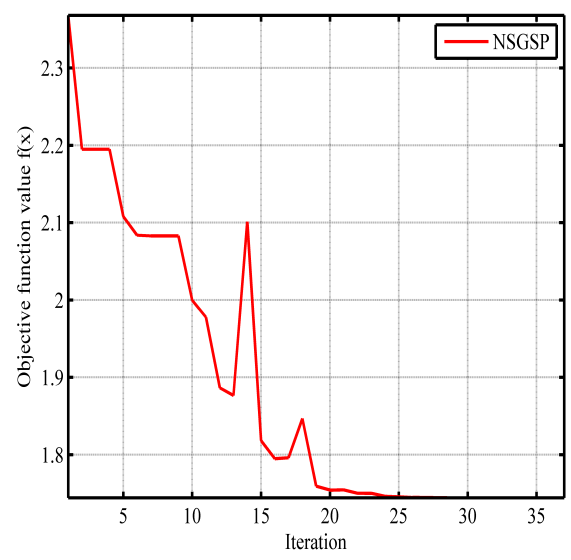

Figure 2: Eason and Fenton gear inertia problem. Minimum is $f(1.74,2.02)=1.74$

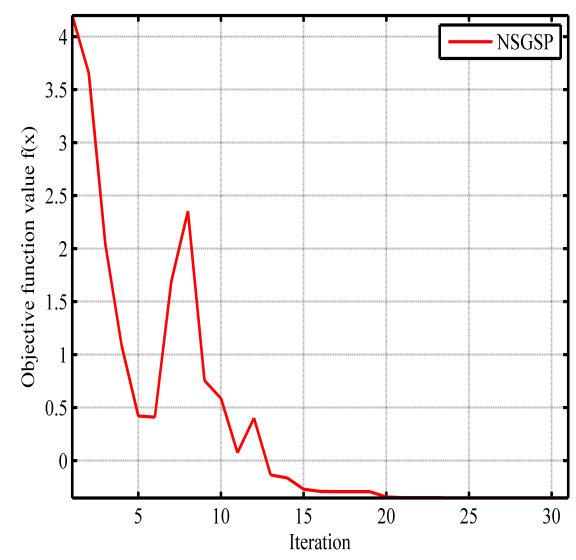

Figure 3: Aluffi-Pentini's function. Minimum is $f(-1.04,0)=-0.35$ 


\begin{tabular}{|c|c|c|c|c|}
\hline Problem & Ref. & Dimension & Optimal design variables $(x)$ & Objective function value $f(x)$ \\
\hline \multirow[t]{5}{*}{1} & Optimal solution & 2 & $0.82288,0.91144$ & 1.3935 \\
\hline & Present method & & $0.82905,0.91255$ & 1.3861 \\
\hline & de KS Lee et al. & & $0.8343,0.9121$ & 1.3770 \\
\hline & Homaifar et al. & & $0.8080,0.8854$ & 1.4339 \\
\hline & Fogel & & $0.8350,0.9125$ & 1.3772 \\
\hline \multirow[t]{4}{*}{2} & Optimal solution & 2 & $2.246826,2.381865$ & 13.59085 \\
\hline & Present study & & $2.2472,2.3467$ & 13.567 \\
\hline & de KS Lee et al. & & $2.246840,2.382136$ & 13.590845 \\
\hline & Deb GA with TS-R & & Unavailable & 13.59085 \\
\hline \multirow[t]{5}{*}{3} & Optimal solution & 5 & $78.0,33.0,29.995,45.0,36.775$ & -30665.500 \\
\hline & Present study & & $80.4786,35.4786,27.0000,37.3972,42.0962$ & -30666 \\
\hline & de KS Lee et al. & & $78.0,33.0,29.995,45.0,36.776$ & -30665.500 \\
\hline & Homaifar et al. & & $80.39,35.07,32.05,40.33,33.34$ & -30005.700 \\
\hline & Coello & & $78.0495,33.007,27.081,45.00,44.94$ & -31020.859 \\
\hline \multirow[t]{5}{*}{4} & Optimal solution & 7 & $2.330499,1.951372,-0.4775414,4.365726,-0.6244870,1.038131,1.594227$ & 680.6300573 \\
\hline & Present study & & $2.2215,1.9406,-0.4361,4.4284,-0.5904,1.1934,1.4673$ & 680.64 \\
\hline & de KS Lee et al. & & $2.32345617,1.951242,-0.448467,4.3619199,-0.630075,1.03866,1.605384$ & 680.6413574 \\
\hline & Michalewicz & & Unavailable & 680.642 \\
\hline & Deb & & Unavailable & 680.63446 \\
\hline \multirow[t]{5}{*}{5} & Optimal solution & 10 & $2.1719,2.3636,8.7739,5.9598,0.9906,1.4305,1.3216,9.8287,8.28009,8.3759$ & 24.30620 \\
\hline & Present study & & $2.1975,2.3053,8.7582,5.0963,1.0535,1.6020,1.3681,9.8739,8.2892,8.2043$ & 24.314 \\
\hline & de KS Lee et al. & & $2.1552,2.4076,8.7780,5.1020,0.9676,1.3576,1.2877,9.8004,8.1878,8.2562$ & 24.3667 \\
\hline & Michalewicz & & Unavailable & 24.690 \\
\hline & Deb & & Unavailable & 24.37248 \\
\hline
\end{tabular}

Table 8 Computational results of optimal solution and optimal value test problems

\begin{tabular}{lllllll}
\hline \multirow{2}{*}{$\begin{array}{l}\text { Problem } \\
\text { no. }\end{array}$} & \multicolumn{5}{c}{ NSGSP method } \\
\cline { 3 - 7 } & Dimension & $\begin{array}{l}\text { Optimal } \\
\text { solution }\end{array}$ & The Best & $\begin{array}{l}\text { The } \\
\text { Worst }\end{array}$ & Mean & SD \\
\hline 1 & 10 & 1.3861 & 1.3861 & 1.3861 & $1.1559 \mathrm{e}-29$ & 1.3861 \\
2 & 2 & 13.592 & 13.567 & 13.532 & $8.32 \mathrm{e}-04$ & 13.59 \\
3 & 5 & -30665 & -30666 & -30666 & $3.0882 \mathrm{e}-05$ & -30666 \\
4 & 7 & 680.62 & 680.64 & 680.68 & $7.4412 \mathrm{e}-05$ & 680.64 \\
5 & 10 & 24.305 & 24.314 & 24.351 & $1.60 \mathrm{e}-05$ & 24.307 \\
\hline
\end{tabular}

Table 9 Computational results of constrained test functions

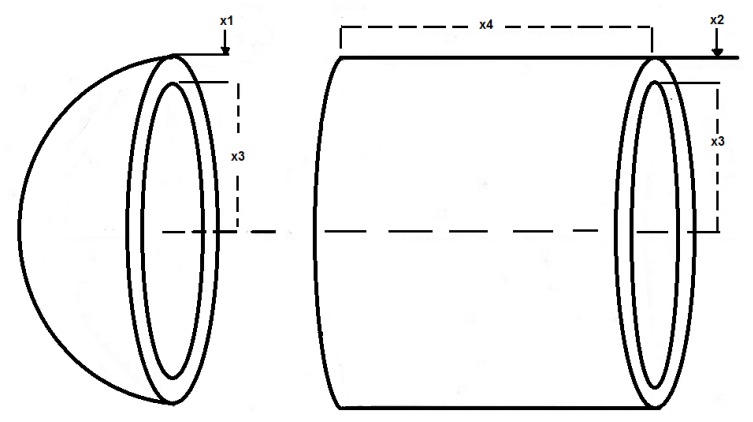

Figure 4: Shematic of the pressure vessel design problem 
Pressure vessel design problem

\begin{tabular}{llll}
\hline The best & The worst & Mean & SD \\
\hline 7195.8 & 7196.0 & 7195.9 & 0.0028 \\
\hline
\end{tabular}

Table 10 Optimal solution of Pressure vessel design problem

\begin{tabular}{lllll}
\hline $\begin{array}{l}\text { Design } \\
\text { variable }\end{array}$ & $\begin{array}{l}\text { Present } \\
\text { method }\end{array}$ & Sandgren & Wu and Chow & $\begin{array}{l}\text { De KS Lee et } \\
\text { al. }\end{array}$ \\
\hline$x_{1}$ & 1.1332 & 1.125 & 1.125 & 1.125 \\
$x_{2}$ & 0.6250 & 0.625 & 0.625 & 0.625 \\
$x_{3}$ & 58.3267 & 48.97 & 58.1978 & 58.2789 \\
$x_{4}$ & 43.4925 & 106.72 & 44.2930 & 43.7549 \\
\hline Cost & 7195.8 & 7980.894 & 7207.494 & 7198.433 \\
\hline
\end{tabular}

Table 11 Comparison results of Pressure vessel design problem

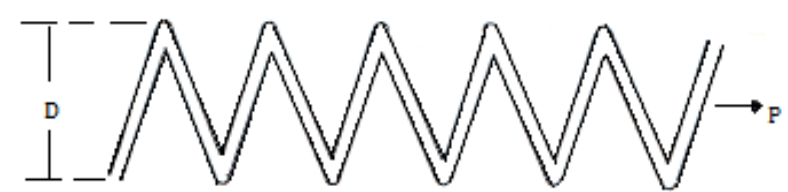

Figure 5: Tension-compression spring design

\begin{tabular}{lllll}
\hline \multicolumn{4}{l}{ Tension-compression spring design } & \\
\hline Parameter & $x_{1}$ & $x_{2}$ & $x_{3}$ & $f(x)$ \\
Value & 0.058324 & 0.604 & 3.7626 & 0.011868 \\
\hline
\end{tabular}

Table 12 Optimal solution of Tension-compression spring design

\begin{tabular}{lllll}
\hline Methods & The best & The worst & Mean & SD \\
\hline NSGSP & 0.011868 & 0.011868 & 0.011868 & $3.0093 \mathrm{e}-36$ \\
GWO & 0.0126660 & 0.0122515 & 0.0121836 & $1.085 \mathrm{e}-05$ \\
CSA & 0.0126652 & 0.0126701 & 0.0127690 & $1.357 \mathrm{e}-06$ \\
CPSO & 0.0126747 & 0.0129240 & 0.0127330 & $5.20 \mathrm{e}-04$ \\
HPSO & 0.0126652 & 0.0127190 & 0.0127072 & $1.58 \mathrm{e}-05$ \\
\hline
\end{tabular}

Table 13 Comparison results of the Tension-compression spring design problem

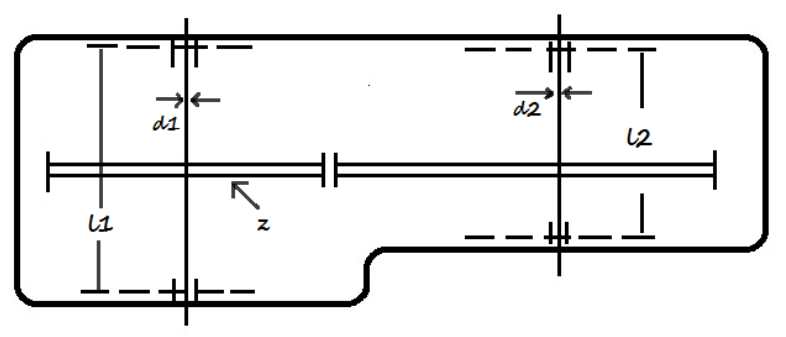

Figure 6: Speed reducer design 


\begin{tabular}{|c|c|c|c|c|c|c|c|}
\hline \multicolumn{8}{|c|}{ Speed reducer design problem } \\
\hline Parameter $x_{1}$ & $x_{2}$ & $x_{3}$ & $x_{4}$ & $x_{5}$ & $x_{6}$ & $x_{7}$ & $f(x)$ \\
\hline 3.600 & 0.700 & 19.069 & 7.300 & 7.800 & 3.1651 & 5.000 & 2995.9 \\
\hline
\end{tabular}

Table 14 Optimal solution of Speed reducer design problem

\begin{tabular}{|c|c|c|c|c|}
\hline Methods & The best & The worst & Mean & SD \\
\hline NSGSP & 2995.9 & 2996.1 & 2996 & 0.0028 \\
\hline $\begin{array}{l}\text { Baykasoglu } \\
\text { and Ozsoydan }\end{array}$ & 2996.37 & 2996.66 & 2996.51 & 0.09 \\
\hline $\begin{array}{l}\text { Mezura-Montes } \\
\text { et al. }\end{array}$ & 2998.01 & 3162.88 & 3056.20 & 49.40 \\
\hline Aguirre et al. & 2996.34 & - & 2996.40 & 0.028 \\
\hline Cagnina et al. & 2996.34 & - & 2996.34 & 0.0 \\
\hline
\end{tabular}

Table 15 Comparison results of Speed reducer design problem

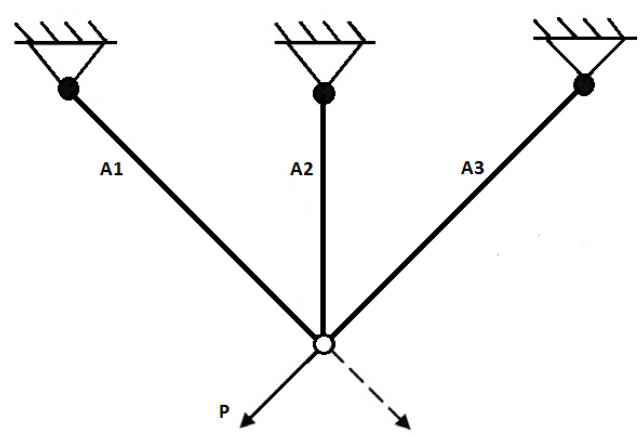

Figure 7: Three bar truss

\begin{tabular}{llll}
\hline \multicolumn{4}{c}{ Three bar Truss } \\
\hline The best & The worst & Mean & SD \\
\hline 263.6789 & 263.6789 & 263.6789 & 0 \\
\hline
\end{tabular}

Table 16 Optimal solution of Three bar Truss

\begin{tabular}{lllllll}
\hline $\begin{array}{l}\text { Design } \\
\text { variable }\end{array}$ & $\begin{array}{l}\text { Present } \\
\text { method }\end{array}$ & $\begin{array}{l}\epsilon D E- \\
P C G A\end{array}$ & $\begin{array}{l}\text { Ray } \\
\text { Saini }\end{array}$ & and & Tsai & $\begin{array}{l}\text { Gandomi et } \\
\text { al. }\end{array}$ \\
\hline \hline$A_{1}$ & 0.7874 & 0.788675 & 0.795 & 0.788 & 0.78867 \\
$A_{2}$ & 0.4076 & 0.408248 & 0.395 & 0.408 & 0.40902 \\
\hline Weight & 263.6789 & 263.89584 & 264.3 & 263.68 & 263.9716 \\
\hline
\end{tabular}

Table 17 Comparison results of Three bar Truss 